\title{
Psychopathologie et sport de haut niveau
}

Psychopathology and high level sport

\author{
M. Salmi*, C. Pichard, E. Jousselin
}

« Département médical » INSEP, 11, avenue du Tremblay, 75012 Paris, France

* Auteur correspondant. Adresse e-mail : meriem.salmi@insep.fr (M. Salmi)

Article paru dans : Science \& sports, 2010, vol. 25, $n^{\circ} 1, p p .1-10$

\section{Résumé}

Contexte. Le sport est largement cité pour ses bénéfices sur la santé (psychologique et physique). Cependant, la pratique intensive des sportifs de haut niveau nécessite une vigilance et un suivi particulier et spécifique sur le versant somatique et psychique. Objectifs.- L'objectif principal est de rendre compte des difficultés psychopathologiques rencontrées par les sportifs de haut niveau de l'Insep. Ce recueil de donnée est réalisé, d'une part, lors du suivi médical longitudinal, à partir des bilans psychologiques obligatoires, et d'autre part, dans le cadre du soin dans la consultation. Matériel et méthodes. Quatre cent soixante-dix sportifs ont passé le bilan psychologique comprenant : un entretien diagnostique structuré (MINI) et un entretien clinique basé sur une grille d'entretien semi-directive. Par ailleurs, 310 sportifs ont été suivis dans une des consultations du suivi psychologique de l’Insep. Résultats. Les troubles anxieux, principalement, l'anxiété généralisée et les épisodes dépressifs majeurs sont les pathologies dominantes. Cela dans le cadre des bilans psychologiques obligatoires mais aussi de la consultation. Conclusion. Les bénéfices du sport sur la santé sont considérables, cependant ce n'est pas un antidote aux problèmes psychopathologiques, notamment lorsqu'il s'agit de sportifs de haut niveau. De plus, on doit souligner qu'à aucun moment le sport ne crée la pathologie, il s'agit plutôt de la rencontre entre une personnalité, un sport et un contexte donné qui crée les conditions d'émergence de la pathologie.

MOTS CLÉS : Psychopathologie ; Sport de haut niveau ; Entraîneurs ; Soins ; Prévention ; Bilans psychologiques

\section{Summary}

Context. Practicing sport regularly provides obvious benefits in health in the broad sense of the term. However, intensive sport activity in particular for high level athletes require vigilance as well as a specific somatic and psychological follow-up. Objectives. The main goal is to show the psychopathological problems encountered by INSEP's high-level athletes. This database has been created firstly with data from mandatory psychological check-ups, made during the longitudinal medical follow-up and secondly with information taken from psycological care consults. Material et methods. Four hundred and seventy athletes have taken the psychological assessment consisting of a structured diagnostical interview (MINI) and a clinical interview based on a semi directing interview scale. The consultation is built on different therapies which exist, indeed 310 sportive have been follow-ups between 2002 and 2008 in one of INSEP's psychological consultation. Results.- Anxiety disorders, principally generalized anxiety generalized, and major depression episodes are the dominant pathologies. This has been found in both mandatory psychological check-ups and consults. Conclusion. The beneficial effects of 
sports on health are extensive, however it is not an antidote for psychopathological problems, especially in the case of high level athletes. Moreover, we should point out that in no case is sport responsible for the pathology, it is the combination of a sport, a personality and a given context which creates the conditions for the start of the pathology.

KEYWORDS : Psychopathology; Sport high level; Coach; care; Prevention

\section{Introduction}

La pratique sportive est largement citée pour son apport bénéfique en termes de santé au sens large du terme. Cependant, la pratique sportive intensive, notamment à propos des sportifs de haut niveau, nécessite une vigilance et un suivi particuliers et spécifiques. Le suivi médical des sportifs de haut niveau, dont la vocation est avant tout préventive, permet entre autres une réduction des risques et une diminution des dommages pour la santé des athlètes.

Cependant et cela même si la santé selon la définition de l'Organisation mondiale de la santé dépasse le modèle médical et englobe la dimension psychique et sociale, la dimension psychologique a longtemps été négligée. L’arrêté du 16 juin 2006 [1] prévoit l'obligation pour les sportifs de haut niveau de réaliser un bilan psychologique. Il s'agit là d'une avancée importante qui engage le suivi non plus uniquement sur un versant somatique, mais bien sur une approche globale où la dimension psychologique reprend une place logique et nécessaire.

Cela étant dit, le suivi psychologique reste le parent pauvre.

Par ailleurs et sans oublier la fonction essentielle et prioritaire du soignant, le bilan psychologique est l'occasion de recueillir un certain nombre de données épidémiologiques. Ces éléments vont permettre de construire et d'élaborer des stratégies de prévention et de soins adaptés et spécifiques aux besoins et demandes des athlètes de haut niveau.

La psychologie clinique et son axe psychopathologique sont relativement récents dans le monde du sport de haut niveau. Les données psychopathologiques sont relativement rares. Nous ferons dans cet article le point sur les premiers éléments psychopathologiques chiffrés recueillis dans le cadre :

- du suivi longitudinal : les bilans psychologiques obligatoires ;

- du soin : la consultation. La demande émane soit du sportif lui même, soit de collègues du département médical ou de l'encadrement sportif (directeur technique national, entraîneurs, coordonnateur...)

\section{Les bilans psychologiques obligatoires}

L’arrêté du 16 juin 2006 précise :

"Deux fois par an chez les sportifs mineurs et une fois par an chez les sportifs majeurs, un bilan psychologique est réalisé, lors d'un entretien spécifique, par un médecin ou par un psychologue sous responsabilité médicale.»

Ce bilan psychologique vise à :

- détecter les difficultés psychopathologiques et des facteurs personnels et familiaux de vulnérabilité et de protection ;

- prévenir des difficultés liées à l'activité sportive intensive ;

- orienter vers une prise en charge si besoin. 


\subsection{Modalités.}

Le bilan psychologique du département médical de l'Insep dure environ une heure et est réalisé par l’un des psychologues cliniciens du service. Il se décline en deux temps.

2.1.1. Un entretien diagnostique structuré qui explore de fac,on standardisée les principaux troubles psychiatriques de l'axe 1 du DSM IV [1] à partir du Mini International Neuropsychiatric Interview, vie passée et actuelle (MINI) [20]

Le MINI (DSM-IV) est un entretien diagnostique structuré d'une durée de passation

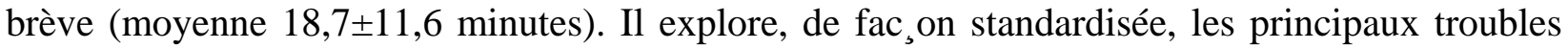
psychiatriques de l'Axe I du DSMIV (American Psychiatric Association, 1994). L’intégralité du MINI contient 120 questions. Les réponses sont dichotomiques. L’outil est divisé en modules, chacun correspondant à une catégorie diagnostique : épisode dépressif majeur (inclus épisode dépressif majeur avec caractéristiques mélancoliques), dysthymie, risque suicidaire, épisode hypomaniaque, trouble panique, agoraphobie, phobie sociale, trouble obsessionnel compulsif, état de stress post-traumatique (option), dépendance alcoolique/ abus d'alcool, troubles liés à une substance (non alcoolique), troubles psychotiques, anorexie mentale, boulimie, anxiété généralisée, trouble de la personnalité antisociale (option). Au début de chacun des modules (à l'exception du module " syndromes psychotiques »), une ou plusieurs questions filtres correspondant aux critères principaux du trouble sont présentées dans un cadre grisé. À la fin de chaque module, une ou plusieurs boites diagnostiques permet (tent) au clinicien d'indiquer si les critères diagnostiques sont atteints.

2.1.2. Un entretien clinique : il s'appuie sur une grille d'entretien semi directive qui prend en compte la spécificité du sportif de haut niveau et la structure INSEP.

Le sport de haut niveau représente l'excellence sportive. Il est reconnu par différents textes législatifs et réglementaires et par la charte du sport de haut niveau qui consacrent l'exemplarité du sportif de haut niveau. Le sport de haut niveau repose sur des critères bien établis qui sont : • la reconnaissance du caractère de haut niveau des disciplines sportives ; • les compétitions de référence : Jeux Olympiques, Championnats du monde et Championnats d'Europe ; • la liste des sportifs de haut niveau ; • les filières d'accès au sport de haut niveau. L'Insep regroupe différentes catégories de sportifs de haut niveau. Depuis 1982, la qualité de sportif de haut niveau s'obtient par l'inscription sur la liste des sportifs de haut niveau arrêtée par le ministère de la Jeunesse et des Sports. Cette inscription s'effectue dans différentes catégories : Élite, Senior, Jeune ou la catégorie Reconversion. Ces catégories ne correspondent pas de façon systématique à des classes d’âges déterminées, ni à des collectifs d’appellation homogène.

2.1.2.1. La catégorie Elite. Peut être inscrit dans cette catégorie, le sportif qui réalise aux jeux Olympiques, aux championnats du monde, aux championnats d'Europe ou dans des compétitions dont la liste est fixée par la Commission nationale du sport de haut niveau, une performance ou obtient un classement significatif, soit à titre individuel, soit en qualité de membre titulaire d'une équipe de France, dans les conditions définies par la Commission nationale du sport de haut niveau. L'inscription dans cette catégorie est valable deux ans. Elle peut être renouvelée dans les mêmes conditions.

2.1.2.2. La catégorie Senior. Peut être inscrit dans cette catégorie, le sportif sélectionné par la fédération délégataire concernée dans une équipe de France pour préparer les compétitions internationales officielles figurant au calendrier des fédérations 
internationales durant l'olympiade en cours et conduisant à la délivrance d'un titre international ou à l'établissement d'un classement international. L'inscription dans cette catégorie est valable un an. Elle peut être renouvelée dans les mêmes conditions.

2.1.2.3. La catégorie Jeune. Peut être inscrit dans la catégorie Jeune le sportif sélectionné dans une équipe de France par la fédération concernée pour préparer les compétitions internationales officielles de sa catégorie d'âge figurant au calendrier des fédérations internationales et conduisant à la délivrance d'un titre international ou à l'établissement d'un classement international. L'inscription dans cette catégorie est valable un an. Elle peut être renouvelée dans les mêmes conditions (Fig.1-3).

La position stratégique de l'Insep, au cœur du Bois de Vincennes, offre un site exceptionnel de 32 hectares aux 850 sportifs de haut niveau - dont 310 internes (99 mineurs) - qui le fréquentent quotidiennement pour leur entraînement au sein de 26 pôles France. Ces sportifs bénéficient de la proximité de tous les moyens de transport pour leurs déplacements en compétition et de la qualité du réseau universitaire et de formation d'Île de France.

L'Insep met en place un accompagnement individualisé des sportifs tant pour leur suivi médical que pour leur formation au travers du double projet, parfois dit projet de vie. Ce double projet sportif et professionnel est une préoccupation fondamentale. Ainsi, parallèlement à la recherche de l'excellence de l'entraînement sportif, l'Insep accorde une importance particulière à l'aide aux sportifs dans la conduite de leur projet de formation et d'insertion [3].

Nous interrogeons l’équilibre de vie du sportif à travers les axes suivants.

2.1.2.4. La sphère personnelle et familiale. Après avoir pris note d'informations générales sur le patient (âge, sexe, niveau d'étude, etc.), ses antécédents personnels (événements particuliers) et familiaux (situation parentale, fratrie, profession des parents).

Nous reviendrons sur sa biographie, l'ensemble des renseignements relatifs au sportif, à sa vie affective, amicale et à son entourage familial.

Si nous avons détecté des troubles, nous réaliserons une anamnèse, permettant de reconstruire l'histoire de la pathologie et des symptômes dans le contexte particulier du patient.

L'anamnèse est un point essentiel du premier entretien, il va permettre d'orienter le psychologue vers un diagnostic et une prise en charge. Dans le même temps, elle va constituer une information de base permettant de donner un sens, une histoire et peut être une explication à la plainte actuelle du patient.

L’anamnèse concerne en premier lieu le patient, sa plainte et son ressenti.

2.1.2.5. La vie sociale. Après avoir recueilli des informations sur les antécédents, nous interrogeons la vie sociale actuelle. L'Insep fonctionne comme un village. Les athlètes y vivent, se logent, s’y restaurent, se forment et s'entraînent ensemble. Au cours de l'entretien, nous accorderons une attention particulière aux jeunes " entrants ». Il est fréquent que cette population présente des troubles de l'adaptation liés à la séparation avec l'environnement familial, scolaire, amical. Par ailleurs, nous interrogeons le vécu de la vie en collectivité qui dans le contexte Insep prend une place particulière car il va souvent, progressivement, occuper tout l'espace de la vie sociale. Par ailleurs, nous savons que maintenir et préserver des liens en dehors du monde sportif est difficile en 
raison des contraintes. Il reste cependant à être vigilant pour préserver cette source d'équilibre.

2.1.2.6. La vie scolaire, universitaire ou professionnelle. Le projet professionnel est l'objet d'attention particulière à l'Insep. Des formules de suivi et d'accompagnement sont aménagées y compris pendant certains déplacements à l'étranger. Il n'en reste pas moins complexe, c'est donc un élément important, notamment pour interroger le vécu de la scolarité, des rythmes de vie, de la capacité d'organisation, de l'adaptation du projet professionnel. . .

2.1.2.7. L'environnement et la vie sportive. C'est un chapitre important, ou dans un premier temps, nous allons recueillir des informations sur la vie sportive : début de la pratique sportive, nombre d'heures d'entraînement par semaine, saisons précédentes et actuelle, biographie sportive personnelle et familiale, arrivée à l’Insep.

Nous prendrons note de la catégorie dans lequel évolue ce sportif (élite, sénior ou junior) ainsi que son palmarès. Nous l'interrogerons sur le vécu des saisons précédentes et actuelles, ses objectifs sportifs, sa gestion du rythme d'entraînement et son vécu des compétitions. Nous privilégierons un temps pour le rapport et les interactions dans le groupe d'entraînement. Nous terminerons par l'évaluation de la qualité et du mode relationnel, des interactions avec son encadrement sportif et plus particulièrement son ou ses entraîneurs.

2.1.2.8. Santé physique. Nous recueillerons l'ensemble des informations concernant les antécédents médicaux (diagnostics et traitements antérieurs), traitements en cours ou passés, sommeil, fatigue, blessures, douleurs. Ces éléments apportent au diagnostic un éclairage fondamental, en effet nombre de sportifs ont du mal à exprimer leurs difficultés pour des raisons contextuelles que nous développerons plus loin (monde des champions, peur d'être exclu. . .). Or nous savons que des plaintes de type somatique : fatigue, douleurs, perturbations du sommeil peuvent masquer ou sont les conséquences des troubles psychopathologiques.

Le versant somatique relève évidemment de l'avis et du suivi du médecin.

\subsubsection{Restitution.}

C'est à l'issue de ce bilan complet que le clinicien proposera ou non en fonction du diagnostic et de l'appréciation clinique, une prise en charge. Nous prenons donc un temps particulier pour expliquer et donner des indications ou conseils au sportif en fonction du résultat du bilan. En dehors, de l'aspect « réglementaire » de la restitution, ce moment est important pour permettre l'orientation éventuelle vers une prise en charge. Dans notre service, le psychologue en charge de la réalisation du bilan est généralement celui qui assurera la prise en charge, en lien avec l'équipe médicale. Il transmet les éléments au médecin responsable du suivi.

Notons toutefois que si le sportif se trouve en situation de crise ou d'urgence, lors de la passation du bilan, nous mettons en place un protocole correspondant adapté.

\subsection{Choix de l'outil}

Nous avons choisi d'utiliser le MINI pour plusieurs raisons. Il répond à l'objectif de détection des troubles psychopathologiques de l'arrêté. Il est reconnu et validé sur le plan international pour son excellente valeur prédictive. 


\subsection{Résultats 470 bilans-diagnostics selon les critères du DSM IV}

Quarante-deux pour cent des sportifs de l'Insep ne présentent pas de troubles psychopathologiques au moment de la passation du bilan.

Cinquante-huit pour cent des sportifs de l'Insep présentent des troubles psychopathologiques au moment de la passation du bilan.

Les bilans interrogent la vie passée et actuelle.

Les résultats sont présentés dans le graphique suivant : Les résultats sont les suivants par ordre décroissant :

- 39 \% - troubles anxieux dont l’anxiété généralisée est le tableau le plus représenté ; • 23 $\%$ - épisode dépressif majeur ;

- $17 \%$ - conduites addictives, principalement l'abus d'alcool ;

- $6 \%$ - troubles des conduites alimentaires (TCA) dont l'anorexie, la boulimie et les troubles non spécifiés ;

- $3 \%$ - troubles psychotiques (Tableau 1 ).

\section{Les soins}

Cette consultation s'adresse aux athlètes et entraîneurs. La population des sportifs y représente de 18 à $24 \%$ de la population de l'Insep (les travaux de rénovation ont réduit le nombre de sportifs passant ainsi de 850 à 550 cette dernière année). Il s'agit soit d'une démarche volontaire, soit de l'orientation d'un collègue du département médical, de l'encadrement sportif (DTN, entraîneur, coordonnateur,. . .), de l'unité d'aide à la performance du DSHN, d'un membre d'un des départements de l'Insep.

On retrouve par ordre décroissant les résultats suivants :

- $49 \%$ - troubles anxieux avec une large majorité pour les tableaux d’anxiété généralisée ;

- $30 \%$ - épisode dépressif majeur

- $8 \%$ - TCA, dont une large prédominance de la boulimie ;

- 7\% - troubles de la personnalité, il s’agit principalement de personnalités « border-line » ou état limite ;

- 2\% - conduites addictives, les substances concernées sont l'alcool et le cannabis ;

- $4 \%$ - tentatives de suicide. Il ne s'agit pas d'une catégorie diagnostique, mais il nous semble utile de le faire apparaître.

\section{Discussion}

\subsection{Limites du MINI}

Deux types de remarques :

Sur le plan général, il s'agit avant tout d'un outil au service du psychologue. Le diagnostic résultant de la passation du MINI ne peut être validé sans l'appréciation du clinicien. Les compétences et le savoir-faire du praticien sont nécessaires pour affiner et corriger le diagnostic du MINI. Il ne permet pas de diagnostiquer les antécédents de troubles, il manque de précision sur les troubles de conduites alimentaires et les conduites addictives. Les réponses dichotomiques ne permettent pas l'évaluation de la sévérité des symptômes, ni de leur retentissement fonctionnel. Par ailleurs, rappelons qu'au moment du bilan, nous ne disposons pas toujours de toutes les informations, ou tous les critères ne sont pas réunis pour poser un diagnostic. Il est souhaitable que cet outil soit administré par un clinicien expérimenté en pathologie psychiatrique. 
L'utilisation régulière de cet outil nous permet aujourd'hui de faire certaines observations, notamment en termes de limites :

- il ne prend pas en compte les spécificités liées au sport de haut niveau ;

- il est applicable à partir de 15 ans, la version kids est en cours de validation. En ce qui nous concerne à l'Insep la population de moins de 15 ans est rare, néanmoins, quelques athlètes sont concernés.

Ces carences sont évidement compensées par la perception clinique du psychologue du service.

\subsection{De l’intérêt des bilans psychologiques}

Le bilan est souvent l'opportunité qui va déclencher la prise de rendez-vous. Le bilan doit être l'occasion d'une démarche pédagogique du praticien. C'est à lui qu'il revient d'expliquer que la psychologie clinique a comme objet d'étude le comportement humain. Il ne s'agit pas uniquement de traiter du versant psychiatrique et ses corollaires péjoratifs souvent associés (fragilité, folie. . .). Il est important de l'aider à comprendre l'intérêt et le bénéfice d’une démarche de prise en charge, sur sa santé psychique et son épanouissement personnel. Dans un souci d'explicitation et de clarification et non pas de polémique, il nous semble important de rappeler que la psychologie clinique n'est pas la psychiatrie, même s’il existe des champs communs, notamment la psychopathologie clinique. En effet, le psychologue clinicien évalue, pose un diagnostic, traite la souffrance psychique en mettant en place les stratégies thérapeutiques adaptées. L'action peut aussi être préventive, l’occasion est alors donnée de proposer des conseils ou un soutien. Mais on peut aussi répondre à des difficultés du quotidien quelle que soit leur origine. La psychologie peut certes prévenir, soigner quand il y a demande, mais elle peut aussi préserver et améliorer la santé psychique, le bien-être, la qualité de vie, développer son potentiel psychologique. En ce sens, le bilan ne remplit pas la seule condition réglementaire et doit être un moment privilégié où le psychologue peut entre autres accompagner ce sportif de haut niveau et veiller à ce qu'il s'engage et préserve une qualité de vie nécessaire à son épanouissement et à son équilibre : on ne naît pas athlète de haut niveau, on le devient. Concrètement, sur 53 \% des propositions de suivi faites à l'issue de la passation du bilan par un des psychologues, $47 \%$ des sportifs sont venus ensuite consulter régulièrement.

Par ailleurs, les données psychopathologiques recueillies dans le cadre du bilan psychologique nous donnent une image de l'état de santé psychologique des sportifs de haut niveau de l'Insep qui nous permet d'élaborer et de mettre en place des stratégies de prévention et de soin adaptées.

\subsection{Diagnostics Bilans psychologiques obligatoires et soins}

En premier lieu, il est très important de lire ces chiffres en tenant compte du contexte des sujets concernés. Ces résultats n’ont aucun sens sans y inclure la dimension du sport de haut niveau. Cette précaution est fondamentale pour comprendre les nuances et subtilités liées à ce monde particulier et hors normes.

\subsubsection{Bilans-Soins}

Dans la colonne bilans athlètes il s'agit des 58 \% des sportifs de l'Insep présentant des troubles psychopathologiques au moment de la passation du bilan, soit $42 \%$ non concernés au moment de la passation du bilan. Sur l'ensemble de ces sportifs, il a été proposé des prises en charge. Nous savons que sur $53 \%$ des propositions de suivi faites à l'issue de la passation du bilan par un des psychologues, $47 \%$ des sportifs sont venus ensuite consulter régulièrement. On peut donc considérer qu'une grande partie de ces athlètes se retrouvent ensuite comptabilisés dans la consultation. Par ailleurs, les sportifs peuvent être suivis avant même le bilan obligatoire. Ce qui 
pourrait expliquer en partie que les écarts ne soient pas frappants. Il reste tout de même un écart sur les conduites addictives, où nous retrouverons plus bas les commentaires. Les 17 \% regroupent principalement les abus d'alcool tel qu’on la retrouve dans la population générale. Cette conduite à risques nécessite une démarche préventive d'information et de sensibilisation. L'orientation sur la consultation et la prise en charge sera indiquée si le comportement persiste ou s'il masque des perturbations psychopathologiques plus importantes.

\subsubsection{Soins athlètes-entraîneurs}

Il nous semblait intéressant de constater et de pouvoir comparer les troubles psychopathologiques rencontrés par les sportifs, et ceux présentés par les entraîneurs. On note que ces derniers sont uniquement concernés par les troubles anxieux et des épisodes dépressifs, à la différence des sportifs qui présentent d'autres troubles psychopathologiques. Cependant, il nous semble utile de préciser que la proportion d'entraîneur est nettement moins importante (environ 10 \%) Ces chiffres sont à lire consciencieusement, nous n'avons pas pour le moment les moyens d'exploiter ces données. Par ailleurs, les difficultés psychologiques rencontrées par les entraîneurs mériteraient un développement ce qui ne correspond pas à l’objet de cet article.

\subsection{Troubles psychopathologiques}

Peu d'études se sont penchées sur cette question de manière globale, citons ici un article de psychopathologie du sport qui tente de faire le point sur les recherches actuelles et les travaux menés par des psychologues ou psychiatres, peu nombreux, sur la problématique sportifs de haut niveau et santé psychologique. La liste de références citées n’est pas exhaustive $[15,2,4,5,13,14,16,17]$.

L'observation clinique de terrain apporte un éclairage global sur ces troubles et permet de répertorier de manière non exhaustive les facteurs favorisant des décompensations notamment sur deux registres : la vie sportive et la vie intime.

\subsubsection{La vie sportive}

La vie sportive et notamment, les situations de compétitions, la concurrence, les rythmes de vie, la qualité de vie (sommeil, fatigue, douleurs, blessures), les déplacements et voyages (décalage horaire. . .), l’intensité et le vécu des entraînements, la qualité et le type de relations avec l'encadrement sportif et plus particulièrement l'entraîneur. . .

\subsubsection{La vie intime}

La vie intime notamment les événements dans l'environnement familial (séparation, difficultés,. . .), la vie collective, l'internat, les amis, les partenaires, les concurrents, la vie scolaire, la vie amoureuse, la vie conjugale. . .

Par ailleurs, deux périodes signifiantes sur le plan des décompensations pour un sportif de haut niveau méritent une attention particulière : la blessure et l'arrêt de carrière.

\subsubsection{La blessure est un moment de fragilité extrême}

La gravité et le temps d'arrêt sont des données importantes dans le vécu, l'intensité et la gravité de la symptomatologie, cependant ce ne sont pas les seuls facteurs Le contexte dans lequel la blessure apparaît ne produira pas les mêmes effets et n'aura pas les mêmes conséquences selon qu'on soit en début de carrière ou fin de carrière, avant une compétition majeure de type Jeux Olympiques ou Championnat du Monde ou une période de relâche. L'environnement sportif, familial, amical intervient de manière importante en fonction du sentiment de soutien apporté, ou 
au contraire, de l'isolement, voire de l'exclusion ressentie. Cependant, la blessure est toujours vécue comme " un drame » même si le temps d'arrêt et de soins est court. Tout se passe comme si le patient vivait un état d'anéantissement. La blessure est vécue comme une atteinte identitaire sur le plan structurel. Il n’existe plus dans ce moment pour lui-même et les autres.

\subsubsection{L'arrêt de carrière est un temps de grande vulnérabilité}

L’intensité et la gravité des symptômes sont variables en fonction de l'environnement, du contexte et du sujet. Schématiquement, trois situations sont possibles : l'arrêt décidé par la fédération (manque de résultats. . .), par blessure, décidé par le sportif.

Quelle que soit la situation, cette période génère des déséquilibres psychiques. Le sportif se retrouve dans une situation de perte identitaire, pour certains un sentiment « d'inexistence ». Un série de pertes : statut social, amis, « famille sportive », habitudes, mode de vie, environnement et le sentiment de ne pas être adapté au "monde ordinaire », Précisons tout de même que de manière générale, plus la carrière est brillante et longue, plus les décompensations sont sévères. Nous ne traiterons pas ici du surentraînement qui nécessiterait un développement particulier. La présence de troubles psychopathologiques n'est pas uniquement liée à la pratique sportive intensive, mais au sportif lui-même, son histoire et sa structure psychique, au contexte et à l'environnement. En fonction de l'influence positive ou négative de ces paramètres, l'évolution de l'athlète sera différente. C'est avant tout une question d'équilibre où tous ces paramètres sont en jeu.

Ce n'est pas le sport qui crée la pathologie, mais la rencontre d'une personne (structure psychique) avec un sport dans un environnement et un contexte donné, notamment de recherche de la performance.

\subsubsection{Les troubles anxieux}

Ce sont les principaux troubles rencontrés par les sportifs. Ils sont classés en fonction des symptômes de la manière suivante : attaque de panique, agoraphobie, trouble panique, phobie spécifique, phobie sociale, trouble obsessionnel compulsif, état de stress post-traumatique, état de stress aigu, anxiété généralisée, trouble anxieux induit par une substance, troubles anxieux non spécifiés.

Les tableaux d'anxiété généralisée y sont principalement représentés. La caractéristique essentielle de l'anxiété généralisée est une anxiété et des soucis excessifs (attente avec appréhension) survenant la plupart du temps durant une période d'au moins six mois et concernant plusieurs événements ou activités. Le sujet éprouve de la difficulté à contrôler ses préoccupations. L'anxiété et les soucis sont accompagnés d'au moins trois symptômes supplémentaires parmi une liste qui comprend agitation, fatigabilité, difficultés de concentration, irritabilité, tension musculaire et perturbation du sommeil.

Une des principales remarques qui découle de cette observation clinique est de constater la capacité anxiogène du sport de haut niveau. Les liens stress et performance ou gestion du stress en compétition sont d'ailleurs largement décrits dans la littérature en psychologie du sport. Il est intéressant de préciser que les professionnels issus de la psychologie du sport n'utilisent pas le même langage, cela tient au fait que nos formations initiales sont différentes. De manière à ne pas créer de confusions, entre autres sur les terminologies utilisées [8], il nous semble important de préciser qu'en ce qui nous concerne, nous faisons référence à la psychologie clinique et nous appuyons entre autres sur l'apport conceptuel du DSMIV. Cependant, la dimension anxiogène n’est pas uniquement liée aux compétitions ainsi que nous l'avons précisé plus haut, elle tient à un ensemble de paramètres. 


\subsection{6. Épisode dépressif majeur}

La caractéristique essentielle de l'épisode dépressif majeur est une humeur dépressive ou une perte d'intérêt ou de plaisir pour presque toutes les activités persistant au moins deux semaines. Le sujet doit de surcroît présenter au moins quatre symptômes supplémentaires compris dans la liste suivante : changement de l'appétit ou du poids, du sommeil et de l'activité psychomotrice ; réduction de l'énergie ; idées de dévalorisation ou de culpabilité ; difficultés à penser, à se concentrer, ou à prendre des décisions ; idées de mort récurrentes, idées suicidaires, plan ou tentatives de suicide. Pour être pris en compte pour un épisode dépressif majeur, un symptôme doit être nouveau ou avoir subi une aggravation évidente par rapport à la situation du sujet avant l'épisode. Les symptômes doivent être présents pratiquement toute la journée, presque tous les jours pendant au moins deux semaines consécutives ; l'épisode doit être accompagné d'une souffrance cliniquement significative ou d'une altération du fonctionnement social, professionnel ou dans d'autres domaines importants. Lors d'épisodes moins sévères, le fonctionnement de certains sujets peut paraître normal, au prix d'efforts notablement accrus.

De nombreux travaux sur les effets de l'activité physique sur la dépression ont été menés, malgré les difficultés méthodologiques ; il semble se dégager un consensus pour la population adulte et plus particulièrement sur les aspects suivants de la dépression : inactivité, isolement, baisse de l'estime de soi, trouble de l'image du corps, inquiétudes somatiques. . . L'activité physique peut jouer un rôle majeur à ce niveau et limiter les inadaptations et donc améliorer la qualité de vie des patients. Un récent rapport de l'Inserm met en évidence entre autres cet aspect [19]. À notre connaissance, nous n’avons aucune étude spécifique sur les corrélations sport de haut niveau et dépression.

On retrouvera globalement les mêmes origines et contextes que pour les troubles anxieux, même s'il existe des particularités significatives en fonction de la structure psychique ou de comorbidités associées.

\subsubsection{Troubles des conduites alimentaires (TCA)}

Le risque de présenter des TCA dans le milieu sportif n'est plus à démontrer. En revanche, apporter des éléments spécifiques ou faire la démonstration que le sport serait à l'origine, favoriserait ou augmenterait le risque n'est toujours pas démontré de manière rigoureuse jusqu'à ce jour. La littérature, à ce propos est contradictoire [19,9,12].

À ce propos, trois réflexions nous semblent importantes : les questionnaires ou outils utilisés dans ces études n'ont pas été standardisés spécifiquement pour les populations d'athlètes ; la dimension sportive manque et doit être intégrée comme une dimension essentielle à l'analyse à la réflexion et aux études ; en dernier lieu, il faudrait aussi pouvoir suivre les sportifs après leur arrêt et ce plusieurs années après l'arrêt pour pouvoir avancer des données sérieuses et fiables. De manière très schématique, pouvoir faire une distinction entre des TCA liés « exclusivement » à la pratique sportive et donc censés être " soignés " à l'arrêt de la pratique sportive et ceux qui perdureront. Ces difficultés d'ordre méthodologique faussent la lecture et donc les résultats.

On distingue dans la littérature certains sports à risque : sports à catégorie de poids (boxe, judo, lutte. . .), sports à caractère esthétique (gymnastique rythmique, gymnastique artistique, patinage artistique, natation synchronisée. ..), sports d'endurance (athlétisme, cyclisme, natation,. . .).

Ce qui dans les faits se traduit par un panel important de sportifs concernés et vient renforcer l'hypothèse selon laquelle tous les sportifs sont susceptibles d'être concernés par cette problématique. Sur le terrain, il nous semble important de nous engager à réfléchir sur : 
- le contexte dans lequel émergent ces troubles (compétitions, période d’adaptation liée à la séparation familiale, ruptures affective ou amoureuse. . .) ;

- les âges de décompensation ;

- les périodes de survenue ;

- la réalité et le vécu différent en fonction des disciplines sportives : sport à catégories de poids (catégories légères ou lourdes), sport à caractère esthétique, sport d'endurance. . . ;

- le vécu psychique singulier.

Ces spécificités doivent nécessairement être prises en compte dans les dispositifs de prévention, d'accompagnement et de soin.

La boulimie est majoritairement représentée dans la population générale, elle l'est aussi dans notre consultation. Elle est plus difficile à détecter que l'anorexie.

Les difficultés diagnostiques sont liées principalement à quatre paramètres :

- dans le monde sportif, les variations de poids sont assez banales ;

- l’évolution et la réussite sportive ne sont pas toujours affectées dans un premier temps. .

.;

- la boulimie est physiquement moins visible ;

- les répercussions sur la santé ne sont pas immédiates.

Il nous semble important d'être attentif à ne pas faire de confusion entre un sportif présentant des TCA réactionnels et provisoires, des TCA chroniques, psychiatriques ou une maigreur «naturelle».

Il nous reste à être vigilant sur certains TCA réactionnels et contextuels (compétitions. . .) pouvant aussi évoluer dans un certain nombre de cas vers une forme grave de la maladie ; d'autant plus qu'il existe souvent une banalisation liée à la pratique sportive intensive.

Les caractéristiques aggravantes sont les pathologies psychiatriques (troubles anxieux, dépressions, trouble de la personnalité, trouble psychotique.), l'apparition précoce, la banalisation, le déni, la durée de l'évolution, l’intensité des régimes, un risque majoré si vomissements, prise de diurétiques, ou autre produit...

Le sport crée un environnement et un contexte psychique et physique particuliers où la question du poids et l'image du corps sont des enjeux « identitaires ».

Compte tenu de la diversité des tableaux cliniques et de la spécificité du sport de haut niveau, il est assez fréquent que les patients ne présentent pas l'ensemble des critères diagnostiques mais que nous ayons des tableaux divers et variés, ce qui rend le diagnostic complexe.

\subsubsection{Troubles de la personnalité et troubles psychotiques}

\subsubsection{Troubles de la personnalité.}

Rappelons en préambule que l'ensemble de la communauté des psychiatres et psychologues s'entendent pour dire que cette catégorie de troubles reste complexe en termes de diagnostic et de catégorisation. Ce qui d'ailleurs donne lieu à nombre de débats, confrontations, " écoles ", polémiques. Le résultat est qu'aucune définition n’a fait consensus sur le plan international. Pour notre part, et pour éviter de relancer le débat, nos références restent la description catégorielle du DSMIV. Un certain nombre de précautions nous sont d'ailleurs indiquées et permettent un diagnostic différentiel.

Ces troubles sont regroupés en trois groupes fondés sur des similarités descriptives. Le groupe A inclut les personnalités paranoïaques, schizoïdes et schizotypiques. Les individus qui ont ces 
personnalités paraissent souvent bizarres ou excentriques. Le groupe B inclut les personnalités antisociales, «borderline », histrioniques et narcissiques. Ces sujets apparaissent souvent sous un jour théâtral, émotif et capricieux. Le groupe $C$ inclut les personnalités évitantes, dépendantes et obsessionnelles compulsives. Les individus ayant ces troubles semblent souvent anxieux et craintifs.

Notons que les auteurs du DSMIV nous précisent que certains troubles de la personnalité, notamment les personnalités antisociales ou borderline, ont tendance à s'estomper ou s'améliorer avec l'âge, ce qui semble moins vrai pour d'autres. Cette observation nous concerne d'autant plus que nous rencontrons principalement des troubles de la personnalité de type border-line (actuel ou passé) ou antisociale (passé). Ils sont d'ailleurs nettement supérieurs dans la consultation que dans les bilans obligatoires.

\subsubsection{Troubles psychotiques.}

Une seule fois depuis 2002, nous avons été amenée à poser un diagnostic de psychose de type schizophrénie. Ce sportif a été amené à arrêter sa pratique sportive de haut niveau. Sa prise en charge a nécessité une hospitalisation et un traitement adapté.

Cependant, nous avons suivi des sportifs présentant des idées délirantes ou hallucinations dans le cadre d’épisodes dépressifs majeurs avec caractéristiques psychotiques.

Il est extrêmement délicat d'avancer des hypothèses à ce stade de nos travaux, nous n'avons pas suffisamment d'éléments et il serait donc risqué de s'aventurer dans des tentatives d'interprétation.

Nous en resterons donc au constat suivant : jusqu’à présent nous n’avons pas détecté (en dehors du sujet cité plus haut) de sportifs de haut niveau présentant un trouble psychotique de type schizophrénique. Tout de même trois questions se posent à la lecture de ces chiffres : ces troubles psychotiques empêcheraient- ils une pratique sportive de haut niveau ? Avons-nous des difficultés à les détecter ? Viennent-ils nous voir en consultation?

\subsubsection{Conduites addictives}

Selon la définition de Goodman, l'addiction se caractérise par l'impossibilité de contrôler un comportement, visant à procurer de plaisir ou soulager un malaise, et continuer la poursuite de ce comportement malgré les conséquences néfastes sur la santé, [10]. Ce comportement regroupe l'usage nocif ou l'abus et la dépendance.

On retrouve principalement chez les athlètes l'usage abusif de substances, dont uniquement l'alcool et le cannabis.

L'abus de substances psychoactives est défini par le DSMIV comme un trouble lié à une substance. Il distingue l’abus et la dépendance.

4.4.9.1. Abus d'alcool (9 \%). La population majoritaire de l'Insep se situe entre l'adolescence et le début de l'âge adulte. Il serait intéressant de pouvoir confronter cette valeur à celle de la consommation des jeunes dans la population générale. Ce comportement d'alcoolisation, qui correspond au « binge drinking » anglo-saxon désigne une alcoolisation massive et ponctuelle [11].

4.4.9.2. Abus de substances (cannabis 1 \%). Il s'agit principalement de cannabis, l'autre produit cité par deux sportifs concernait un solvant (trichloréthylène). Dans la population générale, un tiers des adolescents de 17 ans (22\% des filles et $33 \%$ des garc,ons) déclarent avoir 
consommé du cannabis au cours du dernier mois, ces consommations ayant principalement lieu le week-end [7]. 4.4.9.3. Dépendance à l’alcool (2\%).

4.4.9.3.1. Dépendance aux substances : cannabis (1 \%). Ces chiffres n’apportent pas la confirmation de certaines études qui tendent à démontrer la prévalence de dépendance aux substances dans le milieu sportif notamment l’alcool [6] et autres produits [18].

\section{Conclusion}

Même s’il a été démontré que les bénéfices sur la santé psychique, physique et sociale liés au sport sont considérables et participent d'un équilibre physique et psychique, il n'en reste pas moins que le sport n'est pas un antidote aux problèmes psychopathologiques, d'autant plus lorsqu'il s'agit de sportifs de haut niveau. Les sportifs de haut niveau ne sont pas plus enclins à développer des troubles psychopathologiques, mais ils n’en sont pas épargnés pour autant. Il serait intéressant de mettre en concordance des études sur les problèmes psychopathologiques rencontrés par les élèves des grandes écoles pris dans cette logique de performance.

C'est dans un positionnement psychique et physique, toujours à la limite de la rupture d'équilibre, que se situe l'athlète de haut niveau. Cette situation tout à fait particulière place le sportif au centre d'un paradoxe. Il va devenir dans le même temps extraordinaire et plus fragile. Ce contexte crée le déséquilibre et les conditions de l'émergence de la pathologie. D’autant plus que les sportifs sont nombreux à masquer cette souffrance jusqu'aux décompensations sévères. Le monde des champions a du mal à autoriser les défaillances. Exprimer ses fragilités est particulièrement douloureux et donne le sentiment d'être en danger et vulnérable. Les plaintes sont souvent exprimées sur le versant somatique, seul considéré comme acceptable. Par ailleurs, certains développent une capacité à supporter la souffrance physique et psychique tout à fait impressionnante.

La réalité quotidienne de la consultation reflète des tableaux psychopathologiques divers et variés qui nécessitent le savoir-faire du clinicien. Les diagnostics sont particulièrement complexes dans le milieu sportif de haut niveau, au vu des précédents éléments cités, car le sport occupe une place particulière dans la construction identitaire et l'environnement global du sujet.

Une des questions essentielles du soignant dans l'accompagnement du sportif de haut niveau est bien celle de la question complexe du maintien de l'équilibre.

Il existe trop peu de données sur l'état de santé psychologique des athlètes ; les résultats des bilans et des soins sont pour nous praticiens l'opportunité de réfléchir, d'élaborer des stratégies de prévention, d'accompagnement et de soins adaptés, permettant ainsi la réduction des risques et la diminution des dommages liés à la pratique sportive de haut niveau. Au regard de notre pratique clinique, une attention particulière doit être apportée durant les moments de ruptures liés aux blessures ou à l'arrêt de carrière. Ces périodes présentent un potentiel de risque important en termes de fragilité et de décompensation psychique.

Toutes ces problématiques psychopathologiques s'autorisent des réponses complexes et multifactorielles. Tous les acteurs engagés dans l'accompagnement du sportif ont un rôle et des missions spécifiques en fonction de leurs compétences.

D’autant plus qu’il nous paraît important de souligner qu’à aucun moment le sport ne crée la pathologie, mais qu'il s'agit plutôt de la rencontre entre une personnalité, un sport et un contexte donné qui crée les conditions d’émergence de la pathologie. Nous devons informer, sensibiliser, 
former la communauté sportive sur ces questions. Il s'agit d'une nécessité incontournable pour préserver la santé des athlètes.

Nous devons veiller à établir des collaborations qui ne soient pas une addition d'actions complémentaires, mais une action commune avec pour chacun des compétences spécifiques.

\section{Conflit d'intérêt}

Aucun.

\section{Références}

[1] American Psychiatric Association. Diagnostic and statistical manual of mental disorders. Washington, DC: American Psychiatric Press; 2000.

[2] Bauche P. Les héros sont fatigués. Sport, narcissisme et dépression. Paris: Payot; 2004.

[3] Bouchetal Pellegri F, Leseur V, Debois N. Carrière sportive. Projet de vie. Paris: INSEP Publications; 2006.

[4] Carrier C. Le champion, sa vie, sa mort, Psychanalyse de l'exploit. Paris: Bayard; 2002.

[5] Chamalidis M. Splendeurs et misères des champions. Québec : VLB ;2000.

[6] Choquet M, Ledoux S. Adolescents : Enquête nationale. Paris: Éditions Inserm; 1994.

[7] Consommation de cannabis : niveaux, évolution, géographie. In Cannabis, données essentielles. Saint-Denis : OFDT ; 2006. p. 18-38.

[8] Debois, N., Fleurance, P. Les sources de l'anxiété précompétitive chez les jeunes gymnastes féminines. Rapport de recherche pour le Ministère de la Jeunesse et des Sports 1999.

[9] Filaire E, Rouveix M, Bouget M, Pannafieux C. Prévalence des troubles du comportement alimentaire chez le sportif. Sci Sports 2007;22:135-42.

[10] Goodman A. Addiction: definition and implications. Br J Addict 1990;85(11):1403-8.

[11] Guilbert P, Gautier A. Baromètre santé 2005 Premiers résultats Saint Denis, INPES ; 2006, $176 \mathrm{p}$.

[12] Hagmar M, Hirschberg AL, Berglund L, Berglund B. Special attention to the weight-control strategies employed by olympic athletes striving for leanness is required. Clin J Sports Med 2008;18(1):5-9.

[13] Levêque M. Psychologie du métier d'entraîneur ou l'art d'entraîner les sportifs. Paris: Vuibert; 2005.

[14] Levêque M. Psychologie de l'athlète. Radiographie d'une carrière de sportif de haut niveau. Paris: Vuibert; 2008.

[15] Lincheneau P-M., Franques P., Auriacombe M., Tignol J. Psychopathologie du sport. In : Encyclopédie médico-chirurgicale, 2002 : no 37-887-A-10.

[16] Michel G, Purper-Ouakil D, Leheuzey MF, Mouren-Siméoni MC. Pratiques sportives et corrélats psychopathologiques chez l'enfant et l'adolescent. Neuropsychiatr Enf Adolesc 2003;51(4):179-85.

[17] Michel G, Purper-Ouakil D, Boudin F, Le Heuzey M-F. « Le surentraînement sportif et ses risques psychopathologiques chez l'enfant et l'adolescent ». Synapse 2004;205:23-5.

[18] Nelson TF, Wechsler H. Alcohol and college athletes. Med Sci Sports Exerc 2001;33(1):437.

[19] Rapport Inserm. Activité physique. Contextes et effets sur la santé, 2008. 
[20] Sheehan D, Lecrubier Y, Shenan KH, Amorim P, Janavs J, Weiller E, et al. The MiniInternational Neuropsychiatric Interview (MINI): The development and validation of a Structured Diagnostic Psychiatric Interview for DSM-IV and ICD-10. J Clin Psychiatry 1998;59(20):22-33.

\section{Figures}

Figure 1 Origine de la demande : exemple d'une consultation.

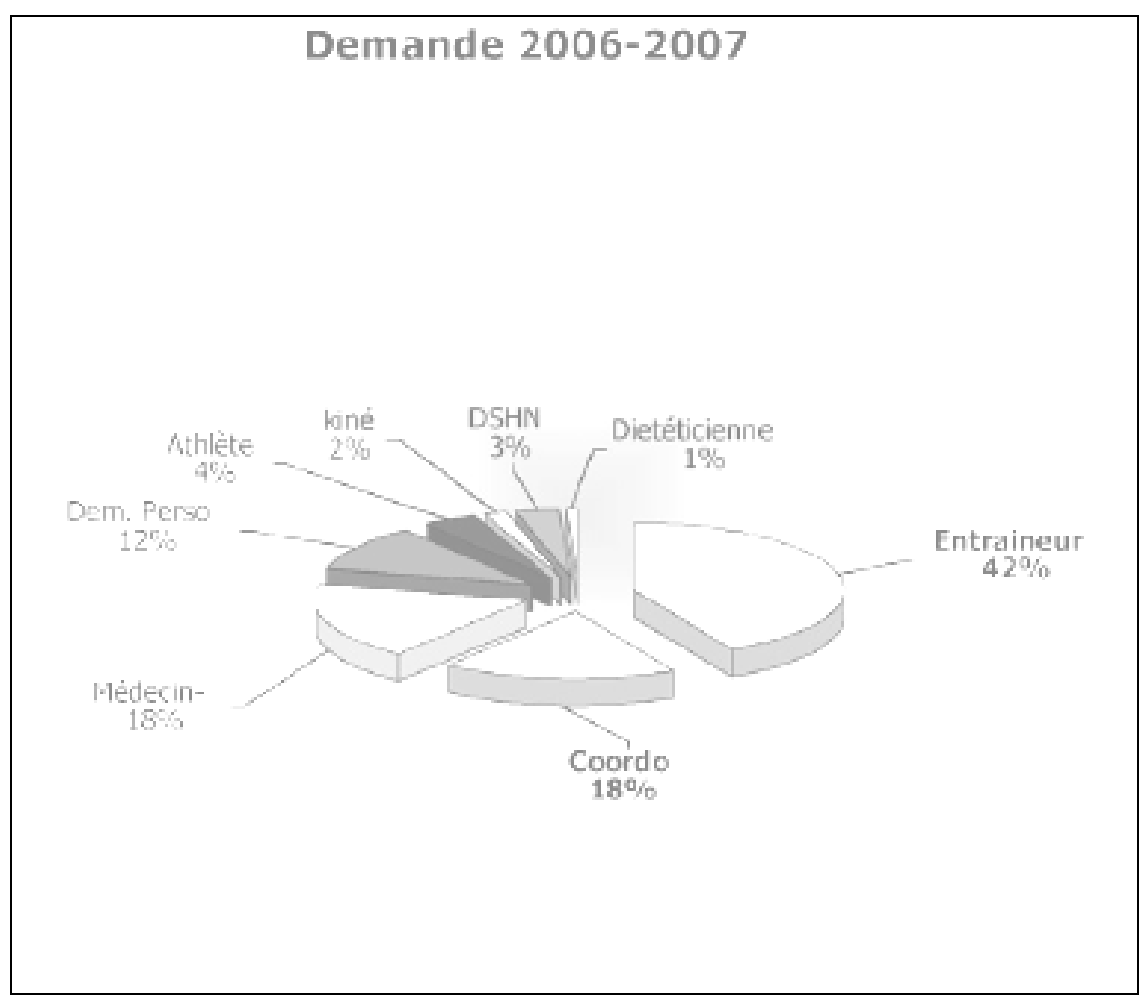


Figure 2 Résultats des bilans.

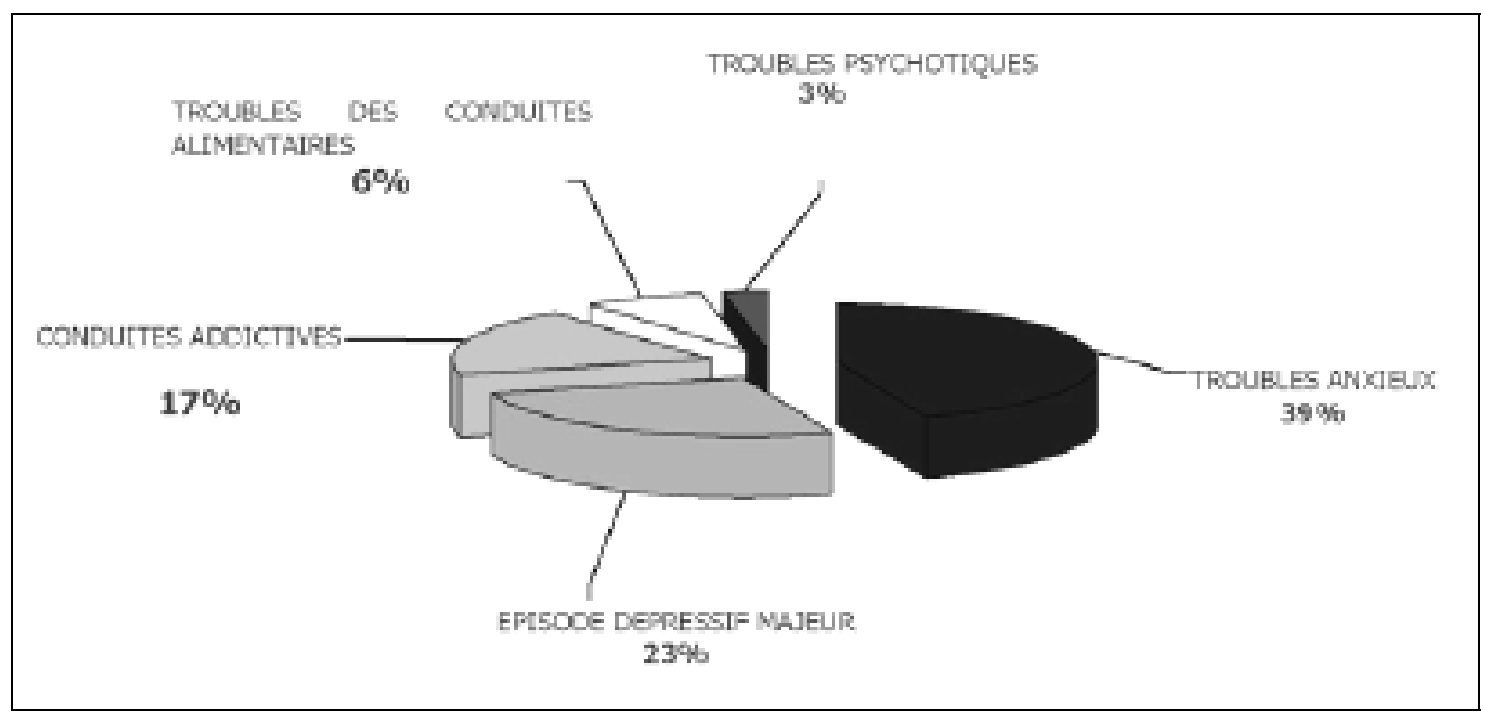

Figure 3 Diagnostics de la même consultation de 2002 à 2008 : 7100 consultations.

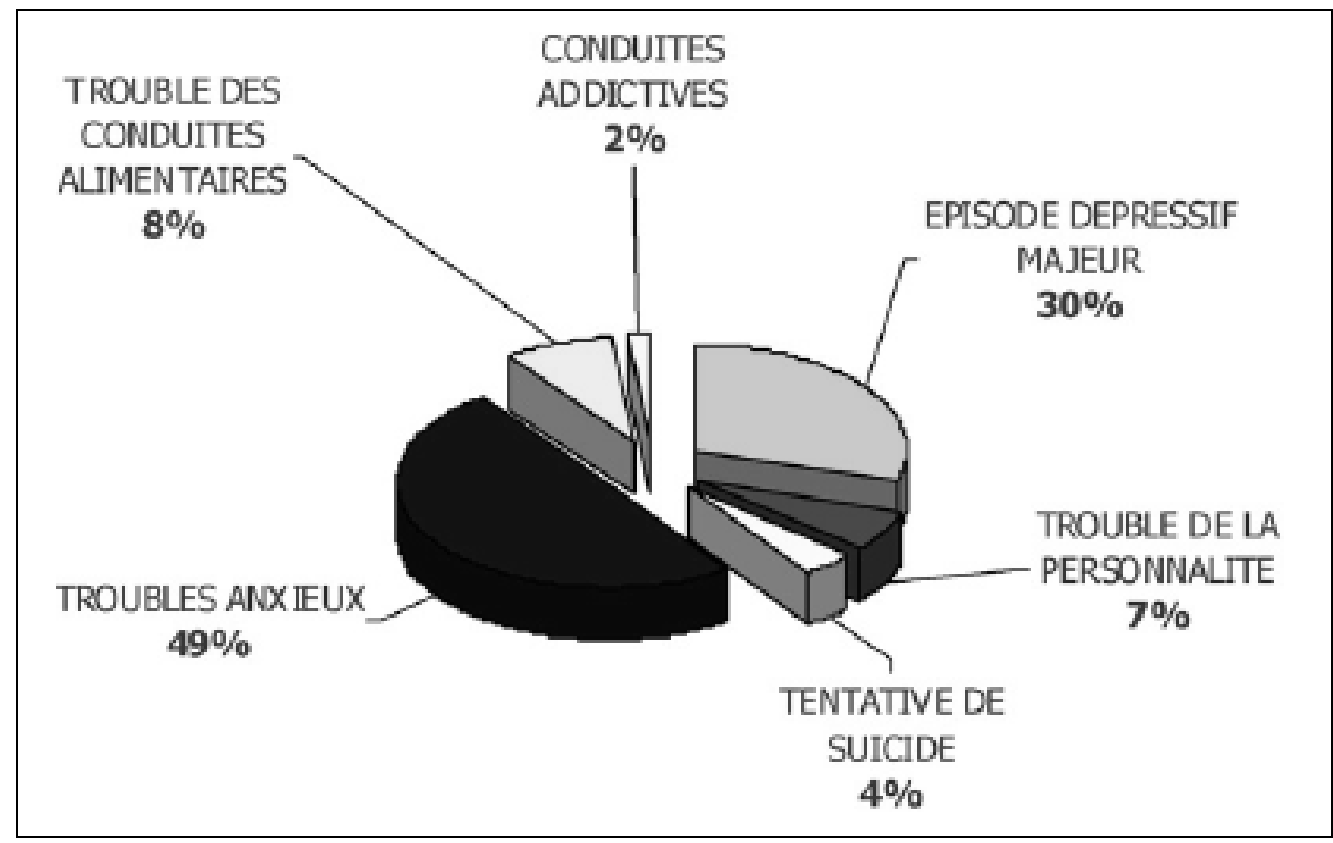


Tableau 1 Diagnostics bilans psychologiques obligatoires et soins athlètes et entraîneurs.

\begin{tabular}{|l|l|l|l|}
\hline Diagnostics & Bilans athlètes (\%) & $\begin{array}{l}\text { Soins Athlètes (même } \\
\text { consultation) (\%) }\end{array}$ & Soins entraîneurs (\%) \\
\hline Troubles anxieux & 39 & 49 & 71 \\
\hline $\begin{array}{l}\text { Épisode dépressif } \\
\text { majeur }\end{array}$ & 23 & 30 & 57 \\
\hline $\begin{array}{l}\text { Troubles des conduites } \\
\text { alimentaires }\end{array}$ & 6 & 8 & 0 \\
\hline $\begin{array}{l}\text { Trouble de la } \\
\text { personnalité } \\
\begin{array}{l}\text { Conduites addictives } \\
\text { (substances) }\end{array}\end{array} 17$ & 7 & 0 \\
\hline
\end{tabular}

